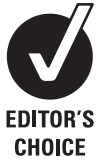

CHOICE

\title{
The joint contribution of sex, age and type of myocardial infarction on hospital mortality following acute myocardial infarction
}

\author{
K P Champney, ${ }^{1}$ P D Frederick, ${ }^{2}$ H Bueno, ${ }^{3}$ S Parashar, ${ }^{1}$ J Foody, ${ }^{4}$ C N B Merz, ${ }^{5}$ \\ J G Canto, ${ }^{6}$ J H Lichtman, ${ }^{7}$ V Vaccarino, ${ }^{1}$ for the NRMI Investigators
}

- An additional figure and tables are published online only at http://heart.bmj.com/content/ vol95/issue11

${ }^{1}$ Emory University School of Medicine, Atlanta, Georgia, USA; ${ }^{2}$ Ovation Research Group, San Francisco, California, USA ${ }^{3}$ Hospital General Universitario "Gregorio Marañón," Madrid, Spain; ${ }^{4}$ Harvard Medical School, Boston, Massachusetts, USA; ${ }^{5}$ Cedars-Sinai Medical Center, Los Angeles, California, USA;

${ }^{6}$ Watson Clinic, Lakeland,

Florida, USA; ${ }^{7}$ Yale University,

New Haven, Connecticut, USA

Correspondence to:

Dr V Vaccarino, Emory University School of Medicine, Department of Medicine, Division of Cardiology, 1256 Briarcliff Road NE, Suite 1 North, Atlanta, GA 30306, USA;

viola.vaccarino@emory.edu

Accepted 23 December 2008

Published Online First

15 January 2009

\section{ABSTRACT}

Objective: Younger, but not older, women have a higher mortality than men of similar age after a myocardial infarction (MI). We sought to determine whether this relationship is true for both ST elevation MI (STEMI) and non-ST elevation MI (NSTEMI).

Design: Retrospective cohort study.

Setting: 1057 USA hospitals participant in the National Registry of Myocardial Infarction between 2000 and 2006.

Patients: 126172 STEMI and 235257 NSTEMI patients. Main outcome measure: Hospital death.

Results: For both STEMI and NSTEMI, the younger the patient's age, the greater the excess mortality risk for women compared with men, while older women fared similarly (STEMI) or better (NSTEMI) than men ( $p<0.0001$ for the age-sex interaction). In STEMI, the unadjusted women-to-men RR was $1.68(95 \% \mathrm{Cl} 1.41$ to 2.01), 1.78 (1.59 to 1.99), 1.45 (1.34 to 1.57$), 1.08$ (1.02 to 1.14 ) and 1.03 (0.98 to 1.07$)$ for age $<50$ years, age 50-59, age 60-69, age 70-79 and age 80-89,

respectively. For NSTEMI, corresponding unadjusted RRs were 1.56 (1.31 to 1.85$), 1.42$ (1.27 to 1.58), 1.17 (1.09 to 1.25$), 0.92$ (0.88 to 0.96 ) and 0.86 (0.83 to 0.89 ).

After adjusting for risk status, the excess risk for younger women compared with men decreased to approximately 15-20\%, while a better survival of older NSTEMI women compared with men persisted.

Conclusions: Sex-related differences in short-term mortality are age-dependent in both STEMI and NSTEMI patients.

Although myocardial infarction (MI) in women occurs more commonly at an older age, it is not solely a disease of elderly women. In the USA, 96000 women younger than 65 years are diagnosed as having acute MI each year, representing $20 \%$ of all acute MI cases in women. ${ }^{1}$ When mortality comparisons between women and men are done separately in younger and older patients, it becomes clear that younger women have a particularly adverse prognosis compared with men, which persists after adjustment for risk factors. ${ }^{2-8}$ In contrast, older women have usually a better survival than older men. ${ }^{2} 6$ 8-12

ST-elevation MI (STEMI) has a worse prognosis in women relative to men compared with other types of acute coronary syndromes. ${ }^{513-16}$ Previous studies looking at the effect of sex on MI mortality have predominantly included non-ST elevation MI (NSTEMI) patients, among whom women are more represented. ${ }^{241617}$ Recent clinical trials of STEMI patients show no significant mortality variation for women compared with men across age. ${ }^{18-20}$ A selection bias due to differential enrolment by gender and the small sample size of younger patients in clinical trials may explain the failure of these trials to demonstrate excess mortality among younger women. Currently it is not known whether younger women drive this excess female mortality in STEMI patients.

Because STEMI and NSTEMI differ in clinical presentation, management and prognostic factors, examining the relationship between sex and mortality according to MI type and patient age should shed light into the potential reasons for sexrelated differences in outcome after MI. The purpose of this study, therefore, was to examine the relationship between sex, age and MI type (STEMI/NSTEMI) on hospital mortality in a contemporary MI sample, and to examine whether observed mortality differences persist after taking into account demographic and clinical patientrelated characteristics.

\section{METHODS}

\section{Patient population}

We conducted a retrospective cohort study of participants in the National Registry of Myocardial Infarction (NRMI). The methods and data collection of NRMI were previously reported. ${ }^{21} 22$ This analysis was based on the two most recent waves, NRMI 4 and 5, which included 794861 patients from 1057 hospitals admitted with MI between July 2000 and February 2006. Patients transferred in or out of the participating hospitals were excluded due to incomplete data regarding presentation, treatment or hospital outcome. Patients with incomplete data regarding age or sex, those $\geqslant 90$ years of age and those with missing levels or levels lower than diagnostic MI levels of CKMB or troponin were also excluded. In addition, patients with prior left bundle branch block (LBBB) were excluded due to the inability to correctly classify MI type. After these exclusions, 361429 patients discharged from participating hospitals between July 2000 and February 2006 were left for analysis.

\section{Definition of STEMI}

Cases with documented ST elevation in more than two leads in the first or subsequent electrocardiogram (ECG), or new LBBB, and abnormal troponin levels or $\mathrm{CKMB}$ at least twice the upper limit of normal were classified as STEMI. 


\section{Definition of NSTEMI}

All remaining cases not classifies as STEMI with abnormal troponin levels or $\mathrm{CKMB}$ at least twice the upper limit of normal were classified as NSTEMI.

\section{Clinical variables}

Each MI type group was classified into five age groups: $<50$ years, 50-59 years, 60-69 years, 70-79 years and 8089 years. Information on all clinical variables was abstracted from the medical records at each hospital. Variables describing demographic characteristics, medical history, cardiovascular risk factors, admission presentation and initial treatments are presented in supplemental tables 1,2 .

\section{Statistical analysis}

First, we examined the frequency and in-hospital mortality of STEMI and NSTEMI for men and women according to age group. Next, we compared baseline demographics, comorbidities and admission presentation between men and women within age groups. To test for trend across the categories of age, the Cochran-Mantel-Haenszel method was used for binary and ordinal variables, and a regression coefficient was calculated and tested for continuous variables. For non-parametric data, the Kruskal-Wallis test was used instead. The female-to-male odds ratio (OR) and 95\% CI for each characteristic were also calculated in each age category. The Breslow-Day statistic was used to test for homogeneity among the ORs produced in each age-specific $2 \times 2$ table. All $p$ values used two-tailed tests of significance. All statistics and calculations used SAS 9.1 (SAS Institute, Cary, North Carolina).

For both STEMI and NSTEMI, we fitted a series of multivariable log-binomial regression models to calculate the relative risk (RR) of in hospital mortality comparing men and women by age category. ${ }^{23}$ When convergence failed, Poisson regression models with robust error variance were used, followed by the methods outlined by Zhang and Yu, in order of decreasing preference. ${ }^{24}$ In these models we also tested the significance of the interaction between age and sex. Each interaction term was tested for significance using the Wald test and type 3 analysis. To assess the impact of demographics, comorbidities and admission presentation on the interaction between age and sex, we fitted a hierarchical series of log-binomial models. The first model included sex as the sole variable and calculated the unadjusted female-to-male relative risk for hospital death in each age category for STEMI, NSTEMI and the overall pooled population. Model 2 adjusted for age and medical history and risk factors; the latter included prior history of cardiovascular disease, renal disease, stroke, diabetes, hypertension, and smoking. Model 3 further adjusted for admission presentation characteristics, which included chest pain at presentation, heart rate, systolic blood pressure, and Killip Class. Model 4 further adjusted for race. Model 5 added primary payor as an independent variable. In a final model, pooling both STEMI and NSTEMI populations, we included all second-order interaction terms and a three-way interaction between sex, age and MI type to determine whether the effect of sex according to age was significantly different in the STEMI vs NSTEMI populations.

\section{RESULTS}

\section{MI prevalence by sex and age}

Of the 361429 patients, 235257 (65\%) were NSTEMI and 126172 (35\%) were STEMI. There were 102081 (43\%) women in the NSTEMI group and 47236 (37\%) women in the STEMI group. Among patients less than 50 years of age, STEMI was four times more common in men than women (79\% vs $21 \%$ ). This difference decreased with increasing age and by age 80, STEMI was more prevalent in women than men. A similar trend, albeit less pronounced, was seen among NSTEMI patients (supplemental fig 1).

\section{Patient characteristics}

Women were older than men, both in the NSTEMI population (median age 76 vs 70 years) and the STEMI population (median age 74 vs 62 years). Women in both MI groups were also more likely than men to have hypertension, diabetes mellitus, congestive heart failure, renal insufficiency, and prior stroke. However, women were less likely to have a prior MI and prior revascularisation, and to present with chest pain (supplemental tables 1,2).

Sex differences in sociodemographic factors and comorbidity were progressively more pronounced with decreasing age in both MI groups. Younger women were much more likely to be of non-white ethnicity, to have Medicaid as their primary insurance and to have a prior stroke, diabetes mellitus, congestive heart failure and renal insufficiency compared with younger men. The increased prevalence of these preadmission characteristics among women, compared with men, decreased with advancing age, and no substantial sex difference was found in these factors among older patients.

Women, particularly younger women, were also less likely than men to present with chest pain and more likely to have tachycardia and hypotension on admission. Among STEMI patients, a substantial proportion, $54 \%$ women and $38 \%$ of men, did not receive reperfusion therapy. Women were less likely to receive reperfusion than men, and this difference was again largest among younger patients (supplemental tables 1,2).

\section{Unadjusted hospital mortality}

As shown in fig 1, MI remains a high-risk condition in this contemporary sample, with high rates of mortality particularly among older patients. Overall, the unadjusted hospital mortality was higher among women than men, and more so in the STEMI population (RR for female sex, $1.64,95 \%$ CI 1.59 to 1.69) than NSTEMI (RR, $1.12,95 \%$ CI 1.09 to 1.15) (fig 1). However, the younger age groups largely drove this sex difference in hospital mortality for both STEMI (RR for female sex in age $<50$ group, $1.68,95 \%$ CI 1.41 to 2.01 ) and NSTEMI (RR for female sex in age $<50$ group, $1.56,95 \%$ CI 1.31 to 1.85 ) (fig 2, table 1). With increasing age, the sex difference in hospital death decreased in both STEMI and NSTEMI ( $p<0.001$ for the interaction between age and sex). Among NSTEMI patients, the sex difference in hospital death actually reversed, with women in the 70-79 and 80-89 age categories having a better survival than men (adjusted RR, $0.92,95 \%$ CI 0.88 to 0.96 and RR 0.86, $95 \%$ CI 0.83 to 0.89 , respectively). The trend of increasing mortality risk for women with decreasing age was similar for both STEMI and NSTEMI, and the three-way interaction term (age, sex and MI type) was not significant $(p=0.77)$.

\section{Multivariable analysis}

Baseline factors, particularly medical comorbidity and presentation characteristics accounted for a substantial portion of the excess risk among young women in both STEMI and NSTEMI patients (fig 2, table 1). In the NSTEMI patients, after adjusting for comorbidity, admission presentation and primary payor, the 


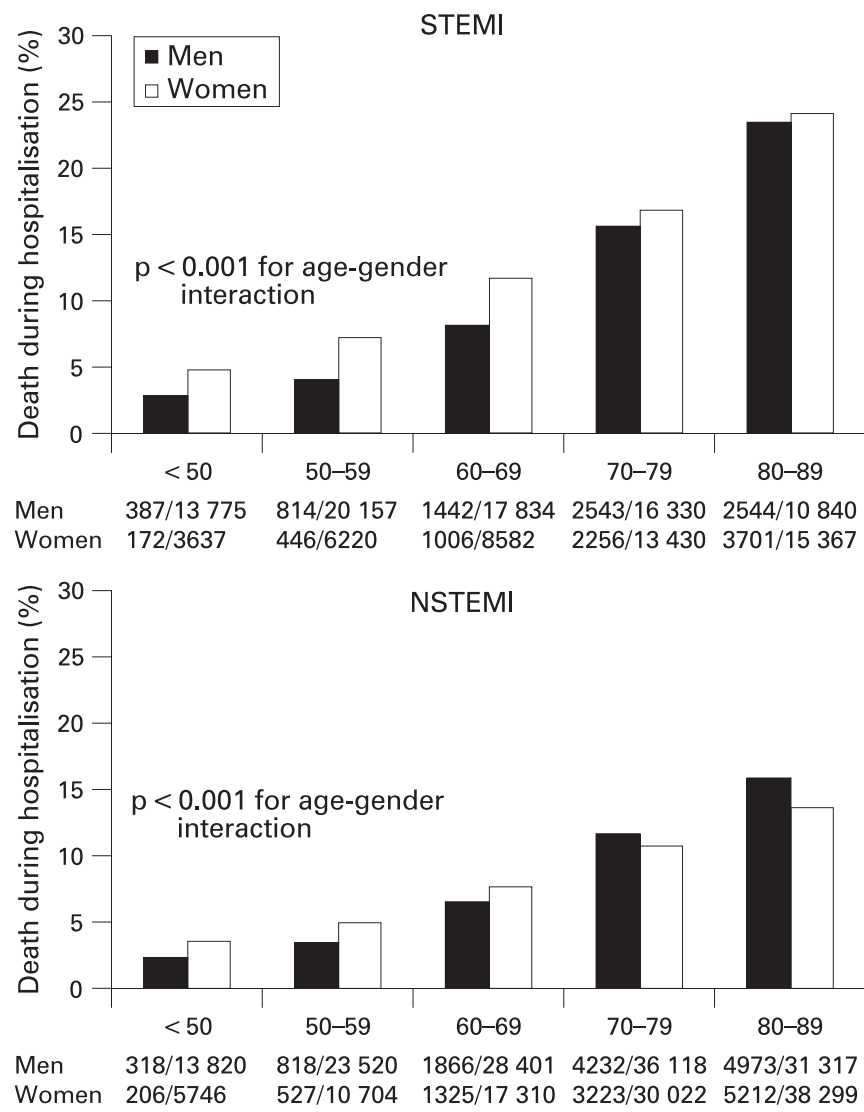

Figure 1 Rates of death during hospitalisation for myocardial infarction among women and men, according to age and myocardial infarction (MI) type. NSTEMI, non-ST elevation MI; STEMI, ST elevation MI.

sex effect decreased substantially and was no longer statistically significant in most age groups below age 70. NSTEMI older women (age 70 year and above), on the other hand, continued to show a significantly lower risk of death compared with men. Similarly, among STEMI patients, the sex effect in the younger age groups was substantially attenuated after adjustment for the same factors, although women below age 70 years maintained an approximately $20 \%$ higher risk of death compared with men in the same age category. The admission presentation variables that mostly explained the sex-related risk in STEMI patients $<50$ years old were absence of chest pain, tachycardia and hypotension. The interaction between sex and age, however, remained highly significant in all adjusted models, while the interaction between sex, age and MI type remained non-significant.

\section{DISCUSSION}

In a large and modern registry of 361429 patients with MI, we found that sex-related differences in short-term mortality are age-dependent in both STEMI and NSTEMI patients. Younger women had a higher hospital mortality than younger men irrespective of MI type, while older women showed no mortality differences (in STEMI) or even a better survival (in NSTEMI) compared with older men. These findings were replicated in a contemporary Swedish MI population where any observed gender differences for in hospital mortality are largely driven by the younger age groups, with women age 51-60 having a significant $78 \%$ increased risk of death. ${ }^{25}$

Younger women had a greater number of preadmission comborbidities and cardiovascular risk factors, more atypical
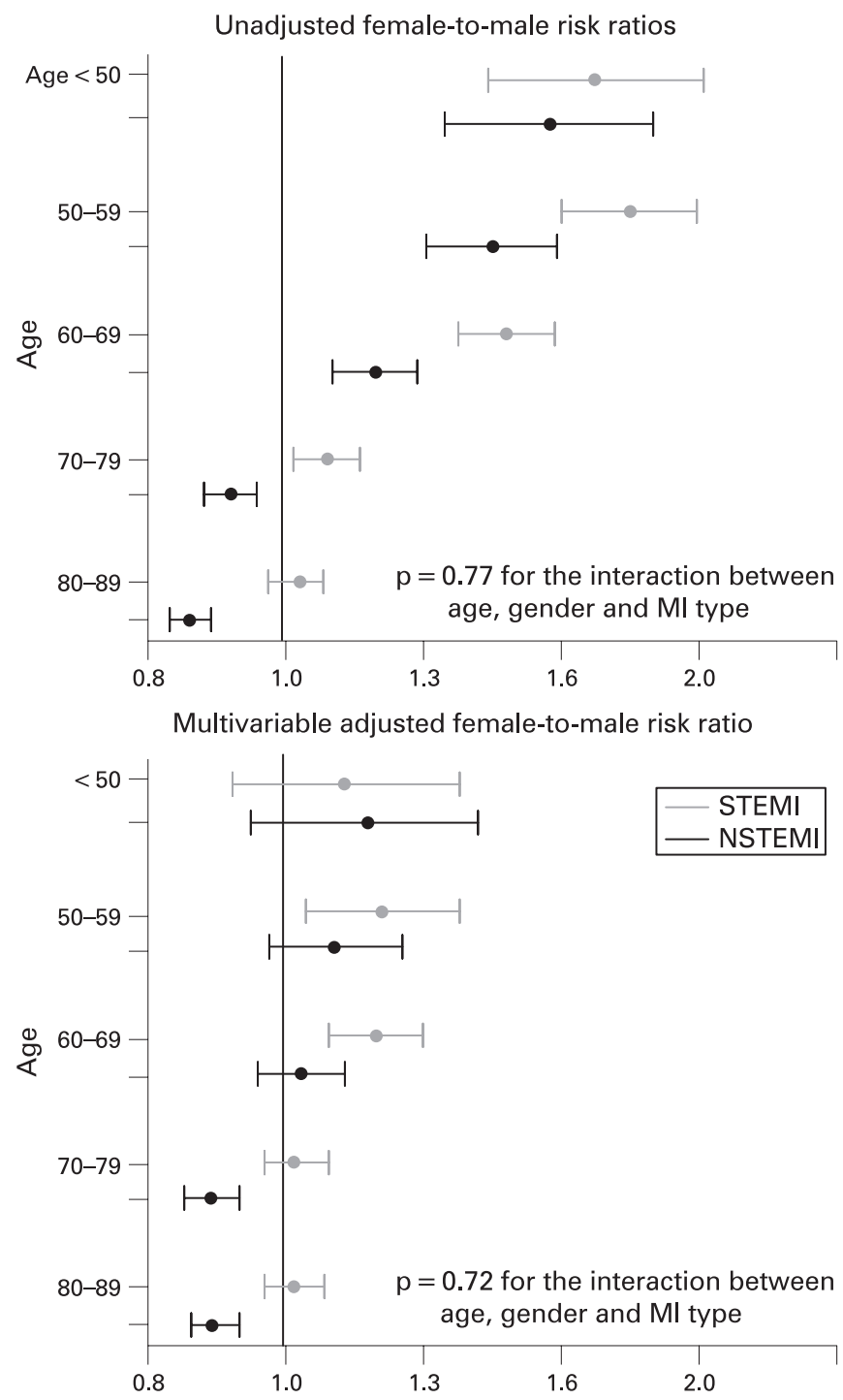

Figure 2 Relative risks for in hospital mortality comparing women with men by age and myocardial infarction (MI) type. The top panel is unadjusted. The bottom panel is adjusted for race, prior cardiovascular disease, renal disease, prior stroke, diabetes, hypertension, smoking, primary payor, body mass index, prehospital delay, admission diagnosis, chest pain, heart rate, systolic blood pressure, Killip class and time of presentation.

symptoms, more adverse presentation signs such as tachycardia and hypotension, and less reperfusion. Each of these findings is attributed to the higher mortality among younger women with MI. Our first risk-adjusted model showed that comorbidities and cardiovascular risk factors account for the majority of sexrelated mortality difference in younger patients. This finding is in accordance with the INTERHEART study which demonstrated that traditional risk factors account for most of the risk of $\mathrm{MI}$ in both younger men and women. ${ }^{26}$ However, mortality remained about $15-20 \%$ higher in younger women than in men of a similar age, particularly in the STEMI population after adjusting for comorbidities and a number of traditional cardiovascular risk factors.

In contrast to the younger women, older women (70 years old and above) had a similar mortality compared with older men in the STEMI population, and significantly lower mortality in the NSTEMI population. This finding is in 
accordance with other studies suggesting that older women fare similarly to, or even better than, older men after an MI, ${ }^{2} 368-12$ with few exceptions..$^{27}$ Our study indicates that when NSTEMI patients are examined separately from STEMI, a survival advantage for older women over men clearly emerges in the NSTEMI group.

In these data, younger women presenting with a MI had more risk factors and were more critically ill when compared with younger men, which in part explained their higher mortality. Since symptomatic coronary heart disease is relatively rare in younger women, a greater number or severity of risk factors may be expected in this group. Studies also suggest that the pathophysiology of coronary heart disease in middleaged or premenopausal women differs from that of older women and men, possibly due to the oestrogen milieu. Younger women are more prone to plaque erosion, whereas older women and men are more prone to plaque rupture. ${ }^{28}$ Atypical symptoms, delayed recognition of heart disease and missed diagnosis of myocardial infarction may also contribute to the higher unadjusted mortality among young women ${ }^{29}$ Consistent with this, in our study absence of chest pain was a major explanatory factor for the higher mortality of younger women compared with men.

A number of possible limitations of our study should be mentioned. This is an observational study; therefore, it cannot prove any causal link for the associations described. Forty-five per cent of our study population was exluded, providing a potential selection bias. However, the vast majority of patients were excluded because of missing cardiac enzymes or cardiac enzymes that were below levels consistent with MI. Thus, most patients were excluded because they probably did not qualify for an MI; these exclusions are unlikely to introduce bias. Another limitation is that we had no information on prehospital deaths. It is possible that the higher in-hospital case fatality of younger women compared with younger men was due to the fact that more men than women with MI die prior to reaching the hospital. Some population-based MI registries support this hypothesis, ${ }^{30}$ but data are not consistent, possibly due to variations and difficulties in ascertaining prehospital causes of death. Information on cholesterol levels is not available in NRMI, and a history of hyperlipidaemia as recorded in the medical records was felt to be unreliable. Therefore, we were unable to adjust for lipid levels in our analysis. Additionally, we did not have any data on long-term outcomes or regarding menopausal status and hormone replacement. Finally, since we had fewer events in the group younger than 50 years than in the older age groups, the CIs for our estimates were larger in the youngest group.

In conclusion, in a contemporary MI patient population we found that sex-related hospital mortality differences are agedependent in both STEMI and NSTEMI patients, with younger women driving the higher mortality of women with MI compared with men. In both MI types, however, any excess risk in younger women is in large part driven by the fact that these women have more risk factors. In contrast, older women with MI are not at increased risk compared with older men, and those with NSTEMI even have a significantly better survival than men.

Funding: KPC: NIH—F32HL82205. VV: NIH—K24HL077506. SP: NIH— K23RR023171. JHL: CDC-1 K01 DP000085. 


\section{REFERENCES}

1. American Heart Association. Heart disease and stroke statistics: 2003 update. Dallas: American Heart Association, 2002.

2. Vaccarino V, Parsons L, Every NR, et al. Sex-based differences in early mortality after myocardial infarction. National Registry of Myocardial Infarction 2 Participants. New Engl J Med 1999;341:217-25.

3. Rosengren A, Spetz CL, Koster M, et al. Sex differences in survival after myocardial infarction in Sweden; data from the Swedish National Acute Myocardial Infarction Register. Eur Heart J 2001;22:314-22.

4. Vaccarino V, Krumholz HM, Yarzebski J, et al. Sex differences in 2-year mortality after hospital discharge for myocardial infarction. Ann Intern Med 2001:134:173-81.

5. Mahon NG, McKenna CJ, Codd MB, et al. Gender differences in the management and outcome of acute myocardial infarction in unselected patients in the thrombolytic era. Am J Cardiol 2000:85:921-6.

6. Kostis JB, Wilson AC, O'Dowd K, et al. Sex differences in the management and long-term outcome of acute myocardial infarction. Circulation 1994;90:1715-30.

7. Andrikopoulos GK, Tzeis SE, Pipilis AG, et al. Younger age potentiates post myocardial infarction survival disadvantage of women. Int J Cardiol 2006;108:320-5.

8. Mukamal KJ, Muller JE, Maclure M, et al. Evaluation of sex-related differences in survival after hospitalization for acute myocardial infarction. Am J Cardiol 2001;88:768-71.

9. Vaccarino V, Krumholz HM, Mendes de Leon CF, et al. Sex differences in survival after myocardial infarction in older adults: a community-based approach [see comment]. J Am Geriatr Soc 1996;44:1174-82.

10. Demirovic J, Blackburn H, McGovern PG, et al. Sex differences in early mortality after acute myocardial infarction (the Minnesota Heart Survey). Am J Cardiol 1995; 75:1096-101

11. Norhammar A, Stenestrand U, Lindback J, et al. Women younger than 65 years with diabetes mellitus are a high-risk group after myocardial infarction: a report from the Swedish Register of Information and Knowledge about Swedish Heart Intensive Care Admission (RIKS-HIA). Heart 2008;94:1565-70.

12. Alfredsson J, Stenestrand U, Wallentin L, et al. Gender differences in management and outcome in non-ST-elevation acute coronary syndrome. Heart 2007:93:1357-62.

13. Malacrida R, Genoni M, Maggioni AP, et al. A comparison of the early outcome of acute myocardial infarction in women and in men. New Engl J Med 1998;338:8-14.

14. Becker RC, Terrin M, Ross R, et al. Comparison of clinical outcomes for women and men after acute myocardial infarction. Ann Intern Med 1994;120:638-45.

15. Weaver WD, White HD, Wilcox RG, et al. Comparisons of characteristics and outcomes among women and men with acute myocardial infarction treated with thrombolytic therapy. JAMA 1996;275:777-82.
16. Hochman JS, Tamis JE, Thompson TD, et al. Sex, clinical presentation, and outcome in patients with acute coronary syndromes. New Engl J Med 1999;341:226-32.

17. Rosengren A, Wallentin L, A KG, et al. Sex, age, and clinical presentation of acute coronary syndromes. Eur Heart J 2004;25:663-70.

18. Mega JL, Morrow DA, Ostor E, et al. Outcomes and optimal antithrombotic therapy in women undergoing gibrinolysis for ST-elevation myocardial infarction. Circulation 2007; 115:2822-8.

19. Reynolds HR, Farkouh ME, Lincoff AM, et al. Impact of female sex on death and bleeding after fibrinolytic treatment of myocardial infarction in GUSTO V. Arch Intern Med 2007;167:2054-60.

20. Motovska Z, Widimsky $\mathrm{P}$, Aschermann $\mathrm{M}$, et al. The impact of gender on outcomes of patients with ST elevation myocardial infarction transported for percutaneous coronary intervention: analysis of the PRAGUE-1 and 2 studies. Heart 2008;94:e5.

21. Rogers WJ, Bowlby LJ, Chandra NC, et al. Treatment of myocardial infarction in the United States (1990 to 1993). Observations from the National Registry of Myocardial Infarction. Circulation 1994;90:2103-14.

22. Every NR, Frederick PD, Robinson M, et al. A comparison of the National Registry of Myocardial Infarction 2 with the Cooperative Cardiovascular Project. J Am Coll Cardiol 1999;33:1886-94.

23. Spiegelman D, Hertzmark E. Easy SAS calculations for risk or prevalence ratios and differences. Am J Epidemiol 2005;162:199-200.

24. Zhang J, Yu KF. What's the relative risk? A method of correcting the odds ratio in cohort studies of common outcomes. JAMA 1998;280:1690-1.

25. Radovanovic D, Erne P, Urban P, et al. Gender differences in management and outcomes in patients with acute coronary syndromes: results on 20290 patients from the AMIS Plus Registry. Heart 2007;93:1369-75.

26. Anand SS, Islam S, Rosengren A, et al. Risk factors for myocardial infarction in women and men: insights from the INTERHEART study. Eur Heart J 2008:29:932-40.

27. Bueno H, Vidan MT, Almazan A, et al. Influence of sex on the short-term outcome of elderly patients with a first acute myocardial infarction. Circulation 1995:92:1133-40.

28. Burke AP, Farb A, Malcom GT, et al. Effect of risk factors on the mechanism of acute thrombosis and sudden coronary death in women. Circulation 1998;97:211016.

29. Pope JH, Aufderheide TP, Ruthazer R, et al. Missed diagnoses of acute cardiac ischemia in the emergency department. New Engl J Med 2000;342:1163-70.

30. Tunstall-Pedoe H, Morrison C, Woodward M, et al. Sex differences in myocardial infarction and coronary deaths in the Scottish MONICA population of Glasgow 1985 to 1991. Presentation, diagnosis, treatment, and 28-day case fatality of 3991 events in men and 1551 events in women. Circulation 1996;93:1981-92. 